\title{
DR3 REGULATION OF APOPTOSIS OF NAIVE T-LYMPHOCYTES IN CHILDREN WITH ACUTE INFECTIOUS MONONUCLEOSIS
}

\author{
Elena Nikolaevna Filatova ${ }^{1 *}$, Elena ViKtorovna Anisenkova ${ }^{1}$, \\ NATALIYA BORISOVNA PRESNYAKOVA ${ }^{1}$ and OLEg Vladimirovich UTKIN ${ }^{1,2}$ \\ ${ }^{1}$ Blokhina Research Institute of Epidemiology and Microbiology, Nizhny Novgorod, Russia \\ ${ }^{2}$ Nizhny Novgorod State Medical Academy, Nizhny Novgorod, Russia
}

(Received: 30 October 2015; accepted: 11 July 2016)

\begin{abstract}
Acute infectious mononucleosis (AIM) is a widespread viral disease that mostly affects children. Development of AIM is accompanied by a change in the ratio of immune cells. This is provided by means of different biological processes including the regulation of apoptosis of naive T-cells. One of the potential regulators of apoptosis of T-lymphocytes is a death receptor 3 (DR3). We have studied the role of DR3 in the regulation of apoptosis of naive $\mathrm{CD}^{+}$(nTh) and $\mathrm{CD}^{+}$(nCTL) T-cells in healthy children and children with AIM. In healthy children as well as in children with AIM, the activation of DR3 is accompanied by inhibition of apoptosis of nTh. In healthy children, the stimulation of DR3 resulted in the increase in apoptosis of nCTL. On the contrary, in children with AIM, the level of apoptosis of nCTL decreased after DR3 activation, which is a positive contribution to the antiviral immune response. In children with AIM, nCTL are characterized by reduced level of apoptosis as compared with healthy children. These results indicate that DR3 can be involved in the reduction of sensitivity of nCTL to apoptosis in children with AIM.
\end{abstract}

Keywords: acute infectious mononucleosis, apoptosis, DR3, naive T-lymphocytes

\section{Introduction}

Acute infectious mononucleosis (AIM) is a widespread viral disease. It is characterized by life-long latent virus persistence and its intermittent reactivation in a host. More than $90 \%$ of the urban population is infected, but an acute form of the disease manifests in only $10 \%$ of them. Most often the disease develops in childhood [1].

*Corresponding author; E-mail: filatova@nniiem.ru 
AIM agents are Herpesviridae, a family of DNA viruses: Epstein-Barr virus (EBV), cytomegalovirus (CMV), and human herpesvirus type 6. They are lymphotropic viruses that can affect the functional state of the cells of the immune system. In particular, they cause anergy of T-lymphocytes and regulate proliferation and apoptosis of immunocompetent cells [2].

It is shown that EBV is accompanied by a significant increase in the number of $\mathrm{CD}^{+}$cytotoxic T-lymphocytes in the peripheral blood [3, 4]. This subpopulation of T-lymphocytes plays an important role in the antiviral immune response both in the lytic and latent phases of AIM [5]. In people with insufficient activation of the $\mathrm{CD}^{+} \mathrm{T}$-cells, the disease progresses fulminantly [6]. The amount of $\mathrm{CD}^{+}$ T-lymphocytes in the peripheral blood in patients with AIM is kept at a constant but at low level. With that $\mathrm{CD} 4^{+} \mathrm{T}$-cells that are activated against virus, antigens are found with high frequency in the peripheral blood of AIM patients [7,8].

A sufficient number of mature immunocompetent cells in peripheral blood are maintained by preservation of the pool of naive T-cells that have proliferative and differentiation potential [9]. In children, the maintenance of homeostasis of T-cells may depend on the thymic output of fresh naive T-lymphocytes, as well as on the proliferation or the death of resting and activated T-cells [10]. In patients with chronic active AIM, a decrease in the content of a common pool of CD4 ${ }^{+}$and $\mathrm{CD}^{+}$T-lymphocytes in the peripheral blood is detected, which is associated with a poor prognosis [11]. Enhanced death of naive T-lymphocytes by apoptosis potentially reduces the effectiveness of immune responses in patients with AIM.

The membrane death receptor 3 (DR3) is expressed on $\mathrm{CD}^{+}$and $\mathrm{CD} 8^{+}$ T-lymphocytes. In vivo DR3 realizes its function through binding to the natural ligand. In vitro DR3 activation may be initiated with addition of monoclonal antibodies or recombinant ligand [12]. DR3 takes part in the activation of apoptosis and proliferation trigger events, as well as in the regulation of the immune response $[13,14]$. At present, DR3 is considered to have mainly a proliferative activity in peripheral T-lymphocytes $[15,16]$. However, this receptor is also involved in negative selection of pre-T-cells in the thymus and initiates the apoptosis following the ligand stimulation [17]. Thus, DR3 signaling may be involved in the regulation of lymphocyte homeostasis by modulating either cell death or lymphocyte activation.

The functional role of DR3 in the immunopathogenesis of AIM has not been studied enough. It is shown that mice that do not express DR3, are more susceptible to CMV infection due to insufficient activation of $\mathrm{CD}^{+} \mathrm{T}$-lymphocytes [18]. In AIM, the participation of DR3 in apoptosis of naive T-cells remains unresolved.

The aim of this study was to estimate the relation of DR3 with apoptosis of naive $\mathrm{CD}^{+}$(nTh) and naive $\mathrm{CD}^{+}$(nCTL) T-lymphocytes in children with AIM. 


\section{Materials and Methods}

\section{Subjects}

The study complies with the Declaration of Helsinki [adopted in June, 1964 (Helsinki, Finland) and revised in October, 2000 (Edinburg, Scotland)] and was approved by the Ethics Committee of Nizhny Novgorod State Medical Academy. Peripheral blood was obtained from children at the age of 9-16 years. Parents or guardians of participants gave written informed consent. Two groups were formed: healthy children $(n=38)$ and children diagnosed with acute infection mononucleosis $(n=34)$.

\section{Cell isolation and purification}

Human peripheral blood mononuclear cells were isolated from fresh whole blood by density gradient centrifugation using Histopaque $\left(\rho=1.077 \mathrm{~g} / \mathrm{cm}^{3}\right.$, Sigma, USA). The subpopulations of $\mathrm{nTh}\left(\mathrm{CD}^{+} \mathrm{CD}^{4} 5 \mathrm{RO}^{-}\right)$and $\mathrm{nCTL}\left(\mathrm{CD}^{+} \mathrm{CD}^{-} 5 \mathrm{RO}^{-}\right)$ were isolated by negative magnetic immunoseparation using commercial kits series EasySep (Stemcell Technologies, UK). The purity of isolated cells was monitored by flow cytometry using a panel of fluorescent-labeled mAb: CD3-PE, CD45ROPE-Cy7, CD45RA-PerCP-Cy5.5, and CD8-APC-eFluor780 (or CD4-APCeFluor780) (eBioscience, USA). We verified that the subpopulations of $\mathrm{nTh}$ and nCTL T-cells were purified to more than 98\% (Figure 1).

\section{Cultivation and activation of naive T-lymphocytes}

The isolated T-cell subpopulations were cultured separately at a concentration of $1 \times 10^{6}$ cells $/ \mathrm{ml}$ in RPMI-1640 medium (PanEco, Russia) and supplemented with $10 \%$ fetal calf bovine serum (PAA Laboratories, Austria) and $2 \mathrm{mM}$ L-glutamine at $37{ }^{\circ} \mathrm{C}$ and $5 \% \mathrm{CO}_{2}$. Specific receptor DR3 activation was performed with mouse anti-human anti-DR3 mAb (clone JD3, isotypeIgGk, eBioscience, USA) in concentration $200 \mathrm{ng} / \mathrm{ml}$. Control naive T-cells were cultivated with addition of phosphate-buffer saline.

\section{Flow cytometry}

All samples were analyzed using a BD FACS Canto II multicolor flow cytometer (Becton, Dickinson and Company, USA). Cytometry setup and tracking beads (BD Biosciences, USA) were used to initialize photomultiplier tubes' settings. 

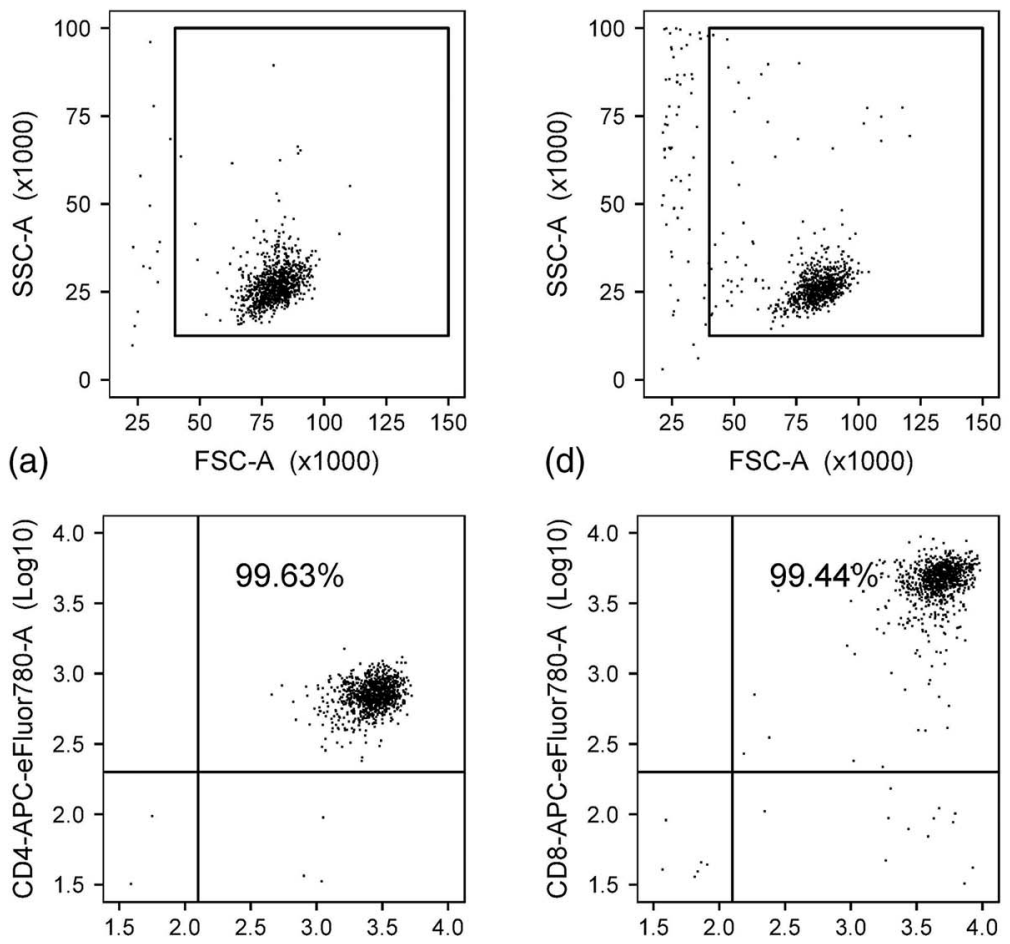

(b) CD3-PE-A $(\log 10)$
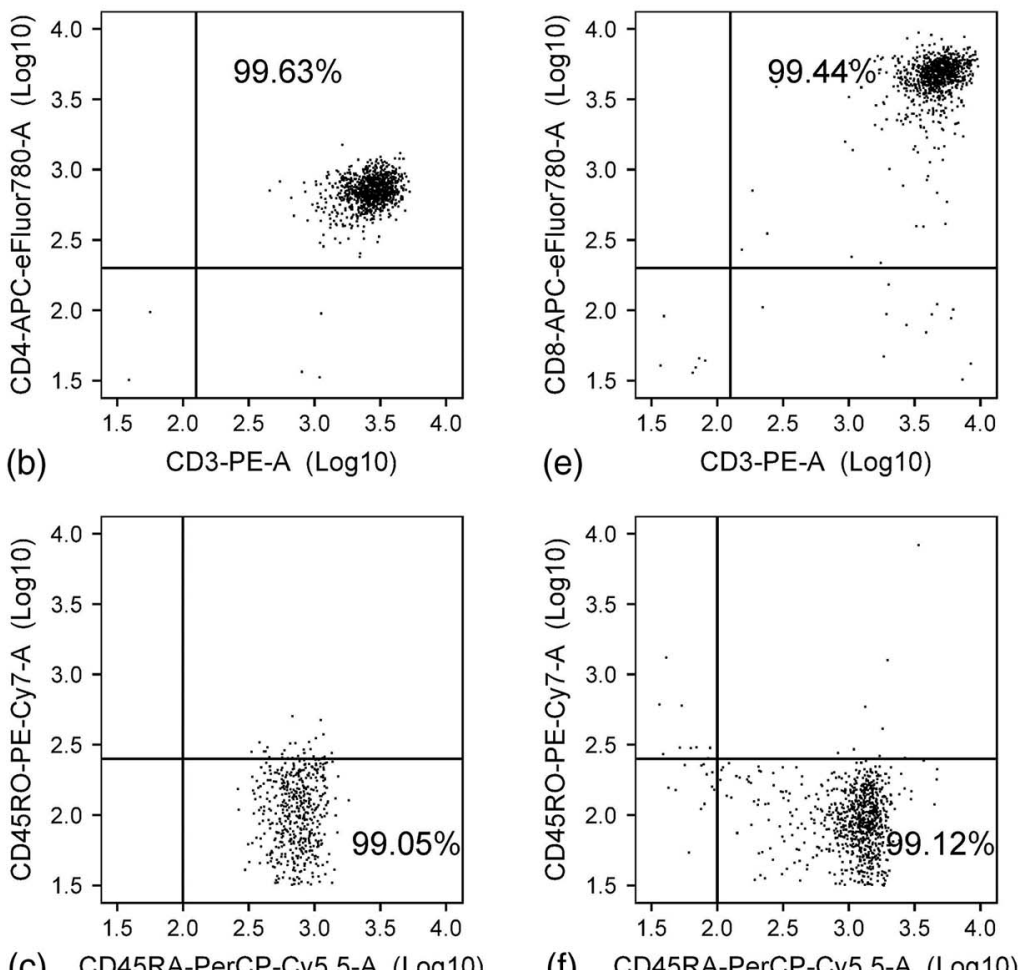

(c) CD45RA-PerCP-Cy5.5-A (Log10)

(f) CD45RA-PerCP-Cy5.5-A (Log10)

Figure 1. Control of naive $\mathrm{CD}^{+}(\mathrm{a}-\mathrm{c})$ and naive $\mathrm{CD}^{+}(\mathrm{d}-\mathrm{f}) \mathrm{T}$-lymphocytes isolation purity by flow cytometry. Based on direct and side scatters, we separated debris and isolated lymphocyte gate (a, d); in an isolated gate, we analyzed the expressions of CD3 and CD4 (b) or CD3 and CD8 (e) markers, the content of $\mathrm{CD}^{+} \mathrm{CD}^{+}$or $\mathrm{CD} 3{ }^{+} \mathrm{CD} 8^{+}$lymphocytes being over $99 \%$; in $\mathrm{CD} 3{ }^{+} \mathrm{CD} 4{ }^{+}$or $\mathrm{CD}^{+} \mathrm{CD}^{+}$lymphocyte pools, we studied the expression of CD45RA and CD45RO markers (c, f),

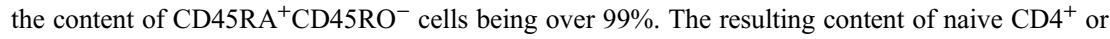
naive $\mathrm{CD}^{+}$T-lymphocytes was over $98 \%$ 
Anti-mouse Ig, k/negative control compensation particles set (BD Biosciences, USA) was used to optimize fluorescence compensation settings. In each sample, 30,000 cells were acquired. For the fluorescent-labeled $\mathrm{mAb}$, the gate settings were established using the relevant isotype controls. All data are acquired electronically in the BD FACS Diva 6.1.3 software (BD Biosciences, USA).

\section{DR3 expression analysis and apoptosis assay}

Apoptosis and DR3 expression assay were performed using flow cytometry. We analyzed freshly isolated nTh, nCTL, and cells after $24 \mathrm{~h}$ of culture with or without anti-DR3 mAb (anti-DR3 mAb treated and control cells). To assess the level of apoptosis, we employed double staining with Annexin V-PE (AV) and 7-aminoactinomycin-D (7AAD) using a commercial kit "PE Annexin V Apoptosis Detection Kit" (BD Biosciences, USA). DR3 expression on the membrane of live and apoptotic naive lymphocytes was analyzed using fluorescently labeled mAb DR3DyLight 488 (Novus Biological, USA). The percentage of live cells and cells in early and late stages of apoptosis, i.e., the percentage of DR3-negative and DR3-positive cells, was calculated using the total number of T-lymphocytes (Figure 2). The density of the membrane expression of DR3 was evaluated based on the mean fluorescence intensity (MFI) of DR3-positive cells.

\section{Data processing}

Two groups of normally distributed data were compared using an independent sample $t$-test or dependent $t$-test for paired samples, and the difference among multiple groups was analyzed using two-way analysis of variance (ANOVA). A Wilcoxon rank-sum test for independent or dependent paired samples was performed to analyze the difference between two groups of non-normally distributed data, and the Friedman test was performed to compare the differences among multiple groups. Mixed-effect linear regression analysis was performed to investigate the relationship between the measured quantitative variables and the treatment of naive T-cells. The $p$ values for multiple comparisons were adjusted using the Holm-Bonferroni correction. $p<0.05$ was considered statistically significant. The presented $p$ values and the significance threshold were two-sided. The difference between two groups was expressed as 95\% confidence interval (CI, unsigned in the text and signed in the illustrations). All statistical analyses were performed using R (version 3.1.1) in the "RStudio 0.98.1098" shell [19, 20] with "stats," "flowCore," "lawstat," "multcomp," and "nlme" packages [21-25]. Illustrations were made using "ggplot2" package [26], and quantitative variables were expressed as median and interquartile range. 

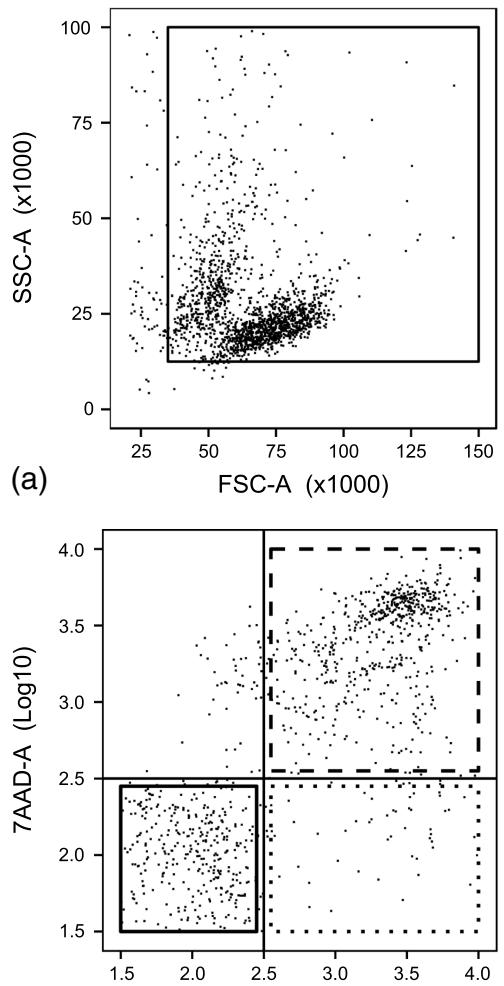

(b) Annexin V-PE-A (Log10)

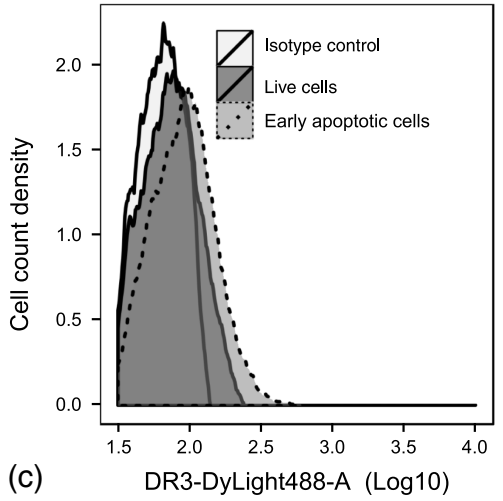

Figure 2. Gating principles when analyzing the apoptosis and expression level of DR3. Lymphocyte gate was separated based on direct and side scatters (a); based on double AV and 7AAD staining, lymphocytes were divided into live cells (solid line), early (dotted line), and late (dash line) apoptotic cells (b); after that gates of live cells and cells in early stage of apoptosis were analyzed separately. In each of them, the percentage of DR3 ${ }^{-}$cells, DR3 ${ }^{+}$cells, and DR3 MFI was determined (c) 


\section{Results}

The content of live and apoptotic freshly isolated naive T-lymphocytes in healthy children and children with AIM

It was shown that in freshly isolated nTh of healthy children, the percentage of live cells was greater $(\mathrm{CI}=10.76-19.79 \%, p<0.001)$ and the percentages of cells in early and late stages of apoptosis were lesser $(\mathrm{CI}=2.34-5.51 \%, p=0.005$ vs. early apoptotic cells; $\mathrm{CI}=5.34-8.08 \%, p=0.016$ vs. late apoptotic cells) than in freshly isolated nCTL. In nTh of children with AIM, the percentage of live cells and cells in early and late stages of apoptosis did not differ from nCTL (Figure 3).

Compared with healthy children, there was no change revealed in the content of freshly isolated live nTh and nTh in early and late stages of apoptosis in children with AIM. In nCTL of children with AIM, the percentage of cells in late stage of apoptosis decreased $(\mathrm{CI}=1.70-11.03 \%, p=0.011)$, and the percentage of live nCTL and nCTL in early stage of apoptosis did not change as compared with healthy children (Figure 3).

DR3 expression on the membrane of freshly isolated live and apoptotic naive T-lymphocytes in healthy children and children with AIM

In general, regardless of the culture conditions in healthy children and children with AIM, the surface density of DR3 expression was greater in naive T-lymphocytes in early stage of apoptosis compared with live cells. In nTh of children with AIM, the rise of DR3 MFI on early apoptotic cells was greater compared with healthy children, especially in freshly isolated cells. Similar results were obtained by comparing the live $\mathrm{nCTL}$ with $\mathrm{nCTL}$ in early stage of apoptosis: in children with AIM, DR3 MFI on the early apoptotic cells increased to a greater extent than in healthy children (Figure 4).

In healthy children, we revealed differences in the expression of DR3 in freshly isolated nTh compared with nCTL. The percentage of live DR3 ${ }^{-}$cells was greater $(\mathrm{CI}=13.72-24.00 \%, p<0.001)$ in $\mathrm{nTh}$ and the percentage of live $\mathrm{DR}^{+}$ cells was lesser $(\mathrm{CI}=1.14-5.33 \%, p=0.009)$ in nTh than in nCTL. The percentage of early apoptotic DR ${ }^{-}$nTh did not differ, and the percentage of early apoptotic DR3 ${ }^{+} \mathrm{nTh}$ decreased $(\mathrm{CI}=1.65-3.30 \%, p<0.001)$ compared with nCTL. DR3 MFI on live and early apoptotic nTh was lesser $(\mathrm{CI}=6.22-13.67$, $p<0.001$ vs. live cells; $\mathrm{CI}=3.17-839.57, p=0.016$ vs. cells in early stage of apoptosis) than on $\mathrm{nCTL}$ (Figure 5).

In children with AIM, there was no difference revealed in DR3 expression between freshly isolated $\mathrm{nTh}$ and $\mathrm{nCTL}$ 


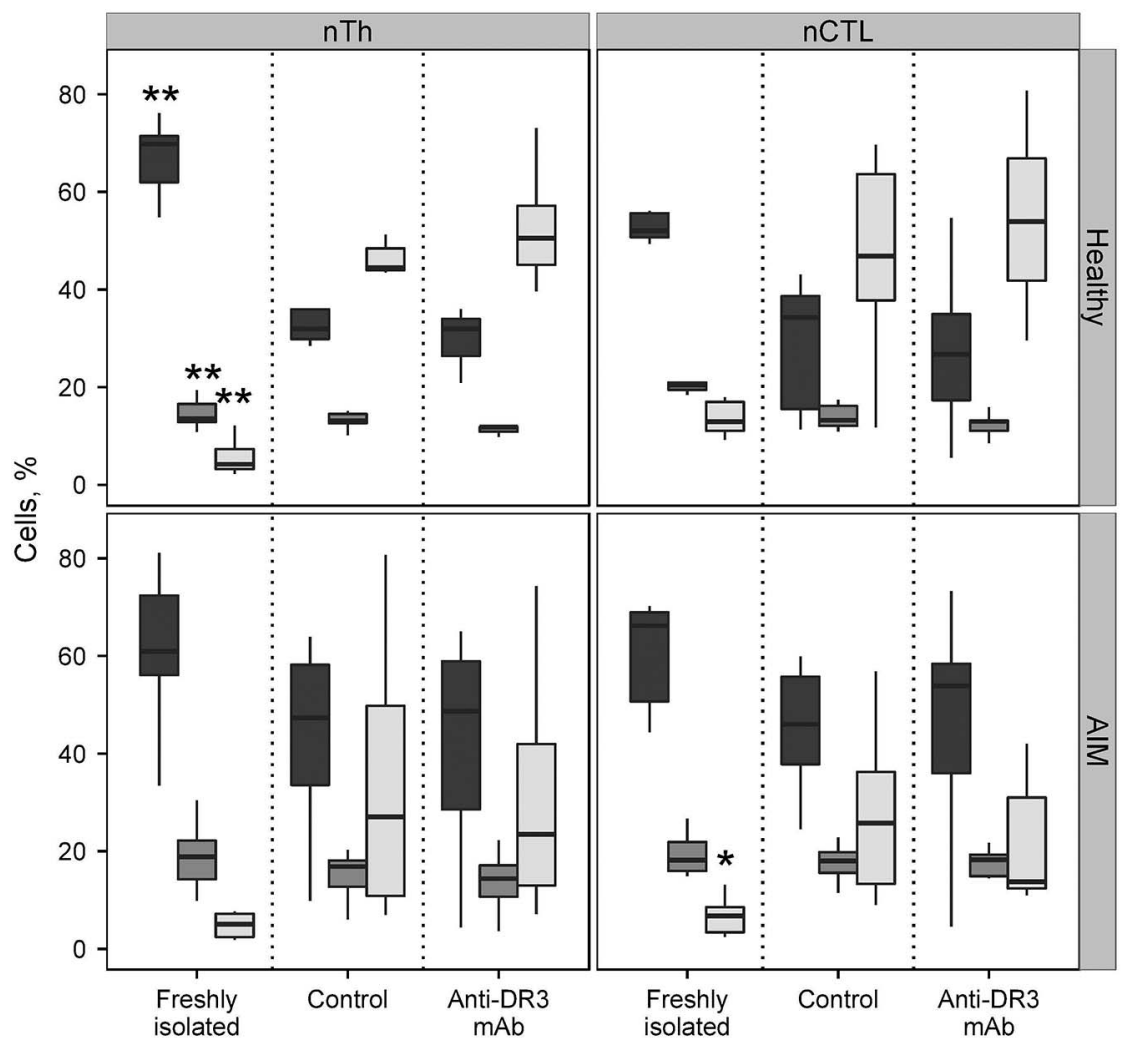

Live cells 官 Early apoptotic cells 官 Late apoptotic cells

Figure 3. The percentage of live and apoptotic naive T-lymphocytes in healthy children and children with AIM.

* indicates statistically significant difference $(p<0.05)$ compared with healthy children; ** indicates statistically significant difference $(p<0.05)$ compared with nCTL

In children with AIM, DR3 MFI was higher $(\mathrm{CI}=110.58-381.66$, $p=0.002$ ) on the membrane of freshly isolated nTh in early stage of apoptosis compared with healthy children. No difference was found in DR3 MFI on the membrane of early apoptotic cells and the percentage of DR $3^{-}$and DR $3^{+}$live and early apoptotic cells when comparing freshly isolated nTh in children with AIM and healthy children (Figure 5).

In children with AIM, the percentage of live freshly isolated DR3- ${ }^{-} \mathrm{nCTL}$ increased $(\mathrm{CI}=2.65-23.73 \%, p=0.030)$ and the percentage of early apoptotic $\mathrm{DR}^{+}$cells decreased $(\mathrm{CI}=0.24-3.36 \%, p=0.037)$ compared with healthy children. DR3 MFI on the membrane of live nCTL was lesser $(\mathrm{CI}=9.02-20.54$, 


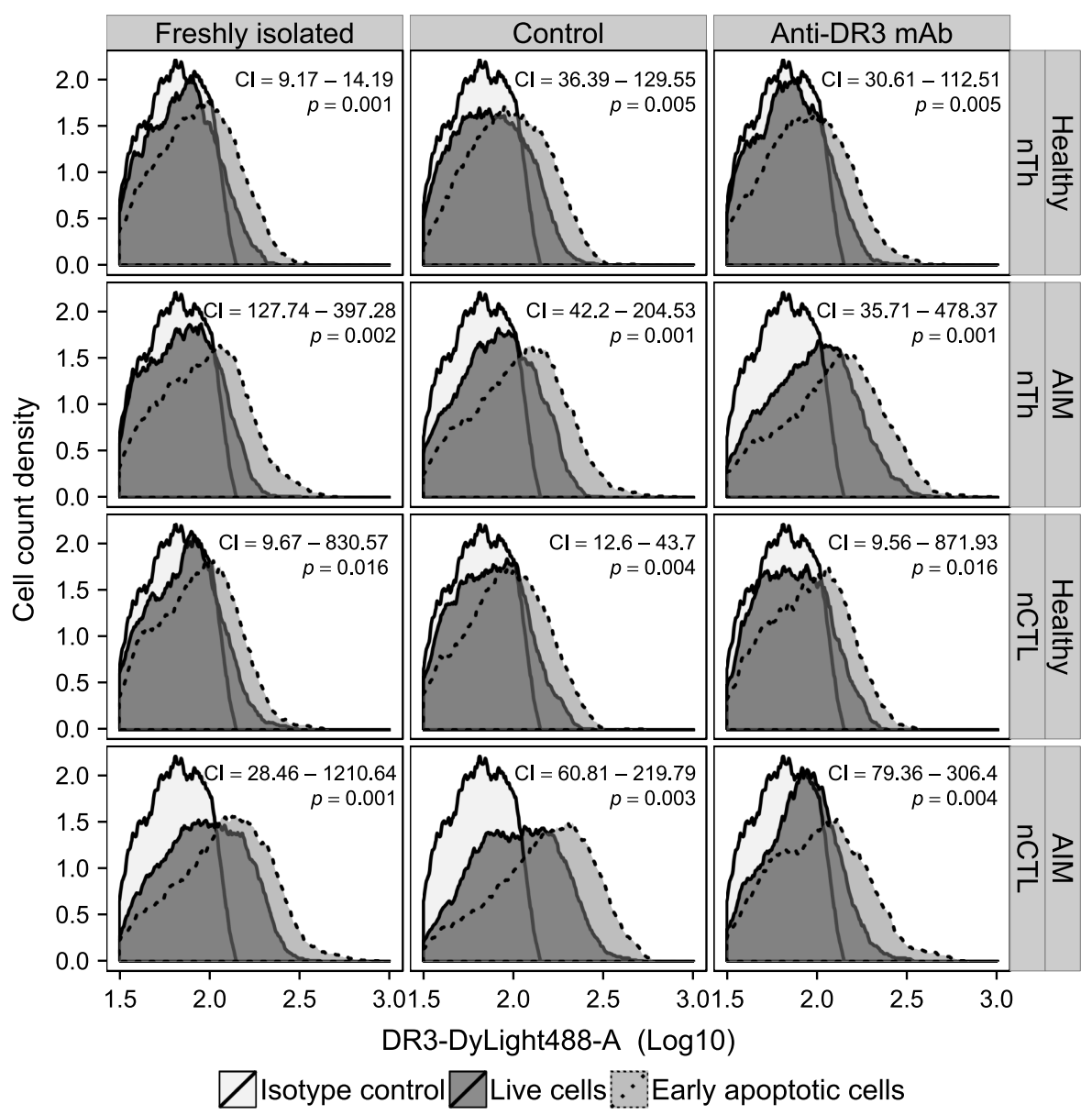

Figure 4. Density plot of DR3 expression on the membrane of live and early apoptotic naive T-lymphocytes in healthy children and children with AIM. The $95 \%$ confidence interval and $p$-value for differences in mean levels of DR3 MFI between live and early apoptotic cells are shown

$p=0.009)$ in children with AIM than in healthy children. No other difference was found in DR3 expression when comparing freshly isolated $\mathrm{nCTL}$ in children with AIM and healthy children (Figure 5).

DR3 impact on the content of live and apoptotic nTh in healthy children and children with AIM

In healthy children with control and anti-DR3 mAb-treated nTh, the percentage of live cells decreased $(\mathrm{CI}=21.94-44.96 \%, p<0.001$ vs. control; 


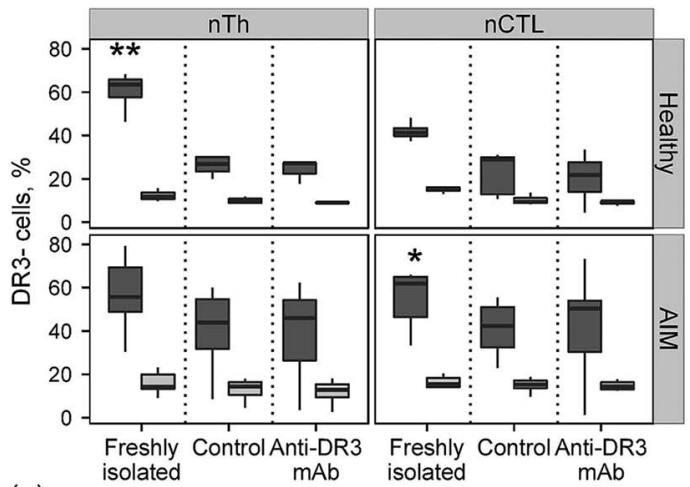

(a)

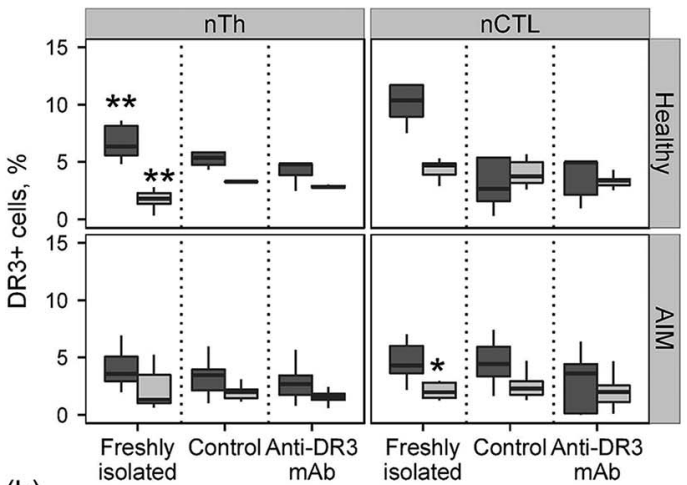

(b)

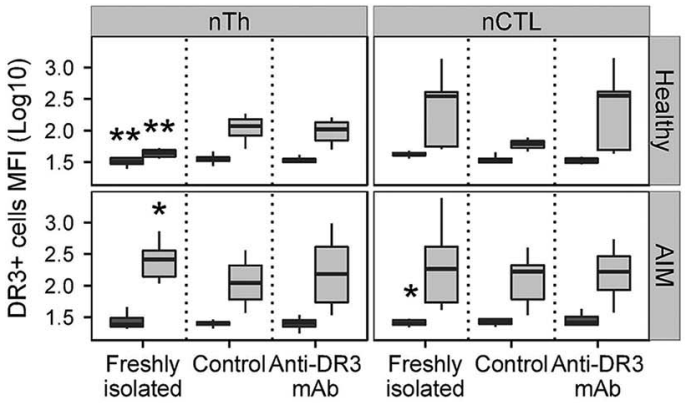

(c)

官Live cells 官Early apoptotic cells

Figure 5. DR3 expression on the membrane of naive T-lymphocytes in healthy children and children with AIM. The percentage of DR3-negative (a), the percentage of DR3-positive (b) naive T-lymphocytes, and DR3 MFI (c) are shown. * indicates statistically significant difference $(p<0.05)$ compared with healthy children; ** indicates statistically significant difference $(p<0.05)$ compared with nCTL 
$\mathrm{CI}=23.29-46.32 \%, p<0.001$ vs. anti-DR3 mAb-treated cells) compared with freshly isolated $\mathrm{nTh}$. The percentage of nTh in early stage of apoptosis did not change but the percentage of cells in late stage of apoptosis increased $(\mathrm{CI}=27.16$ $49.07 \%, p<0.001$ vs. control; $\mathrm{CI}=33.88-55.78 \%, p<0.001$ vs. anti-DR3 mAbtreated cells) in both cases compared with freshly isolated cells (Figure 6a).

In control and anti-DR3 $\mathrm{mAb}$-treated $\mathrm{nTh}$, the decrease in the percentage of live cells was a result of the decrease in the percentage of live DR3 ${ }^{-}$cells $(\mathrm{CI}=22.66-42.46 \%, p<0.001$ vs. control; $\mathrm{CI}=23.61-43.40 \%, p<0.001$ vs. anti-DR3 mAb-treated cells), because the percentage of live DR $3^{+} \mathrm{nTh}$ did not change as compared with freshly isolated cells (Figure 5a and b). Only in control $\mathrm{nTh}$, DR3 MFI on the membrane of live cells increased $(\mathrm{CI}=1.01-5.76, p=$ 0.003) compared with freshly isolated cells; in anti-DR3 mAb-treated cells, DR3 MFI rate did not change (Figure 6c).

While the percentage of nTh in early stage of apoptosis did not change in antiDR3 mAb-treated cells, the percentage of early apoptotic DR3 ${ }^{-} \mathrm{nTh}$ decreased $(\mathrm{CI}=0.91-9.10 \%, p=0.012)$ compared with freshly isolated cells. No such change was found in control cells (Figure 6b). We did not find the difference in the percentage of $\mathrm{DR} 3^{+} \mathrm{nTh}$ in early stage of apoptosis both in control and anti-DR3 $\mathrm{mAb}$-treated cells compared with freshly isolated $\mathrm{nTh}$. However, in both cases, DR3 MFI on the membrane of early apoptotic cells was greater $(\mathrm{CI}=39.85-109.49$, $p<0.001$ vs. control; $\mathrm{CI}=26.18-95.73, p<0.001$ vs. anti-DR3 mAb-treated cells) than in freshly isolated $\mathrm{nTh}$ (Figure $6 \mathrm{~b}$ and $\mathrm{c}$ ).

In children with AIM in control and anti-DR3 mAb-treated nTh, the percentage of live cells decreased $(\mathrm{CI}=3.14-34.34 \%, p=0.010$ vs. control; $\mathrm{CI}=4.90-36.11 \%, p=0.006$ vs. anti-DR3 mAb-treated cells) compared with freshly isolated cells. In control nTh, the percentage of cells in early stage of apoptosis did not change and that in anti-DR3 mAb-treated cells decreased $(\mathrm{CI}=$ $0.65-8.96 \%, p=0.020$ ) compared with freshly isolated cells. Both in control and anti-DR3 mAb-treated $\mathrm{nTh}$, the percentage of cells in late stage of apoptosis increased $(\mathrm{CI}=8.89-43.32 \%, p=0.001$ vs. control; $\mathrm{CI}=12.62-47.05 \%, p<0.001$ vs. anti-DR3 mAb-treated cells) compared with freshly isolated cells (Figure 6a).

While the percentage of live nTh decreased, both in control and anti-DR3 $\mathrm{mAb}$-treated cells, the percentage of live $\mathrm{DR} 3^{-}$cells decreased $(\mathrm{CI}=1.70$ $33.41 \%, p=0.019$ vs. control; $\mathrm{CI}=3.09-34.81 \%, p=0.015$ vs. anti-DR3 $\mathrm{mAb}$-treated cells) and the percentage of live DR $3^{+}$cells did not change compared with freshly isolated cells (Figure 6b). No change was found in DR3 MFI on the membrane of live cells in both cases (Figure 6c).

In children with AIM, no difference was found in DR3 expression pattern in early apoptotic cells between control and freshly isolated nTh. In anti-DR3 mAbtreated cells, the percentage of nTh in early stage of apoptosis decreased as a result 


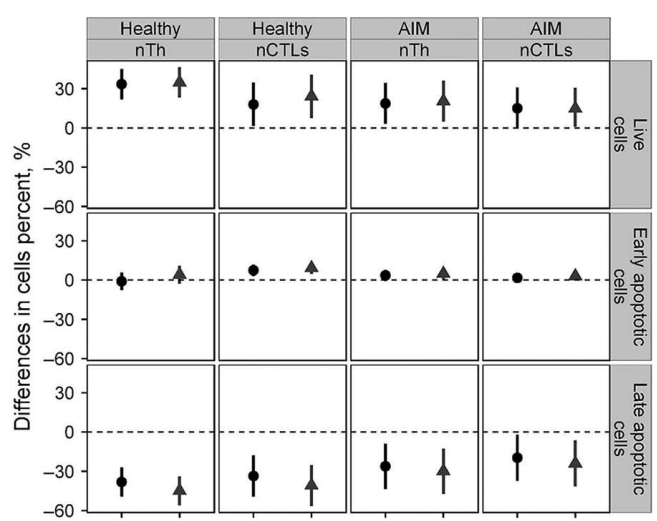

(a)

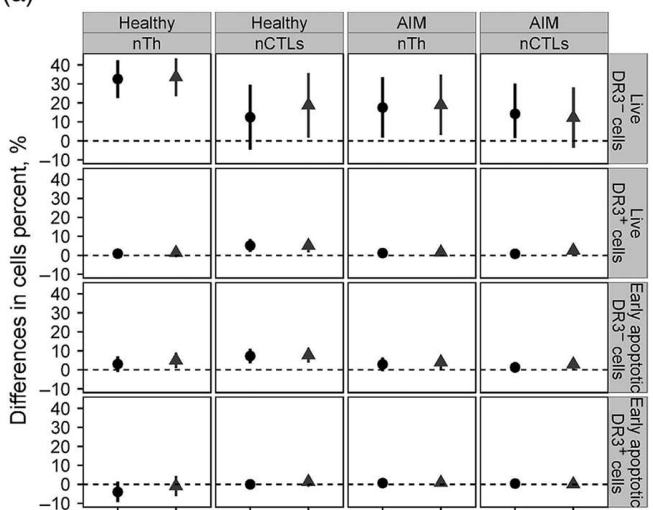

(b)

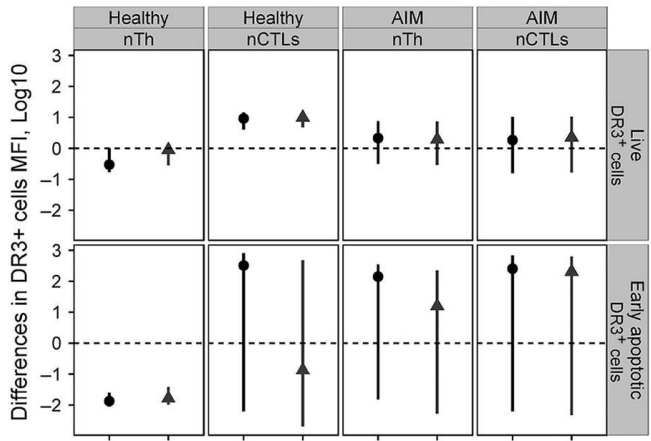

(c)

$$
\begin{aligned}
& \text { - Freshly isolated - } \\
& \text { Control }
\end{aligned}
$$

Figure 6. Differences in mean levels of the percentage of live and apoptotic naive T-cells (a), the percentage of DR3-negative and DR3-positive naive T-cells (b), and DR3 MFI (c) 95\% family-wise confidence level is shown 
of decreased percentage of early apoptotic DR $3^{-}$cells $(\mathrm{CI}=0.48-7.51 \%, p=$ 0.023 ) compared with freshly isolated cells. The percentage of DR $3^{+} \mathrm{nTh}$ in early stage of apoptosis and DR3 MFI on their membrane did not change simultaneously (Figure $6 \mathrm{~b}$ and $\mathrm{c}$ ).

DR3 impact on the content of live and apoptotic $n C T L$ in healthy children and children with AIM

In healthy children in control and anti-DR3 mAb-treated cells, the percentage of live nCTL decreased $(\mathrm{CI}=1.42-34.50 \%, p=0.022$ vs. control; $\mathrm{CI}=7.61-$ $40.69 \%, p=0.002$ vs. anti-DR3 mAb-treated cells) compared with freshly isolated cells. The percentage of $\mathrm{nCTL}$ in early stage of apoptosis also decreased $(\mathrm{CI}=$ $3.02-11.78 \%, p<0.001$ vs. control; $\mathrm{CI}=4.76-13.52 \%, p<0.001$ vs. anti-DR3 $\mathrm{mAb}$-treated cells), while the percentage of $\mathrm{nCTL}$ in late stage of apoptosis increased $(\mathrm{CI}=18.02-49.09 \%, p<0.001$ vs. control; $\mathrm{CI}=25.23-56.31 \%, p<$ 0.001 vs. anti-DR3 mAb-treated cells) in both cases compared with freshly isolated $\mathrm{nCTL}$ (Figure 6a).

In control nCTL, the decrease in the percentage of live cells was a result of the decrease in the percentage of live DR $3^{+}$cells $(\mathrm{CI}=1.81-8.52 \%, p<0.001)$, while the percentage of live DR3 ${ }^{-}$cells did not change compared with freshly isolated $\mathrm{nCTL}$. In anti-DR3 $\mathrm{mAb}$-treated $\mathrm{nCTL}$, there was the decrease in the percentage of both live DR3 ${ }^{-}$and DR $3^{+}$cells $(\mathrm{CI}=1.80-35.72 \%, p=0.029$ vs. live DR3 ${ }^{-}$cells; $\mathrm{CI}=1.69-8.40 \%, p<0.001$ vs. live DR3 ${ }^{+}$cells) compared with freshly isolated nCTL (Figure 6b). Both in control and anti-DR3 mAb-treated nCTL, DR3 MFI on the membrane of live cells was lesser $(\mathrm{CI}=4.08-14.31, p<$ 0.001 vs. control; $\mathrm{CI}=4.78-15.01, p<0.001$ vs. anti-DR3 mAb-treated cells) than on the membrane of freshly isolated nCTL (Figure 6b and c).

In both control and anti-DR3 mAb-treated nCTL of healthy children, the decrease in the percentage of early apoptotic cells was a result of the decrease in the percentage of DR3 ${ }^{-}$cells in early stage of apoptosis $(\mathrm{CI}=3.97-11.45 \%, p<$ 0.001 vs. control; $\mathrm{CI}=3.53-11.01 \%, p<0.001$ vs. anti-DR3 mAb-treated cells) compared with freshly isolated nCTL. No change was found in the percentage of $\mathrm{DR}^{+}{ }^{+} \mathrm{nCTL}$ in early stage of apoptosis or DR3 MFI on their membrane in both cases compared with freshly isolated cells (Figure $6 \mathrm{~b}$ and c).

In control and anti-DR3 mAb-treated cells of children with AIM, the percentage of live nCTL decreased $(\mathrm{CI}=0.72-30.78 \%, p=0.046$ vs. control; $\mathrm{CI}=0.96-30.53 \%, p=0.046$ vs. anti-DR3 mAb-treated cells) compared with freshly isolated cells. The percentage of nCTL did not change in control and decreased in anti-DR3 $\mathrm{mAb}$-treated cells $(\mathrm{CI}=0.20-5.87 \%, p=0.047)$ comparing 
with freshly isolated cells. In both cases, the percentage of nCTL in late stage of apoptosis was greater $(\mathrm{CI}=2.08-37.12 \%, p=0.018$ vs. control; $\mathrm{CI}=6.44-$ $41.49 \%, p=0.004 \mathrm{vs}$. anti-DR3 mAb-treated cells) than in freshly isolated nCTL (Figure 6a).

In control nCTL, the decrease in the percentage of live cells was the result of the decrease in the percentage of live DR3 ${ }^{-}$cells $(\mathrm{CI}=1.50-30.03 \%, p=0.043)$, while the percentage of live DR3 + cells did not differ as compared with freshly isolated nCTL. On the contrary, in anti-DR3 mAb-treated cells, the percentage of live DR3 ${ }^{-}$cells did not change and the percentage of live DR3 ${ }^{+}$cells decreased $(\mathrm{CI}=0.12-4.95 \%, p=0.042)$ compared with freshly isolated nCTL. DR3 MFI on the membrane of live cells did not differ in both cases compared with freshly isolated $\mathrm{nCTL}$ (Figure $6 \mathrm{~b}$ and $\mathrm{c}$ ).

In control nCTL of children with AIM, the percentage of DR3 ${ }^{-}$and DR3 ${ }^{+}$ cells in early stage of apoptosis did not differ from freshly isolated nCTL. In antiDR3 mAb-treated nCTL, the decrease in the percentage of cells in early stage of apoptosis was the result of the decrease in the percentage of early apoptotic DR3 ${ }^{-}$ cells $(\mathrm{CI}=0.10-5.67 \%, p=0.045)$, while the percentage of early apoptotic DR ${ }^{+}$ cells did not change as compared with freshly isolated nCTL. In both cases, no change was found in DR3 MFI on the membrane of nCTL in early stage of apoptosis compared with freshly isolated cells (Figure 6b and c).

\section{Discussion}

In the literature data, the question of DR3 expression in T-cell subpopulations still remains unclear. Jones et al. could not detect DR3 expression on resting human Th, but activation with stimulatory anti-CD3 antibodies led to a marked upregulation of DR3. At the same time, Twohig et al. detected DR3 expression on both murine Th and CTLs, and expression of the receptor was greater on Th compared with CTLs $[18,27]$. It was shown that resting human $\mathrm{CD}^{+}$or $\mathrm{CD}^{+}$ T-cells express very little LARD-1 mRNA. LARD-1 is a full-length form of DR3 that has a transmembrane domain. Resting cells mainly express mRNA that codes soluble forms of the protein, but LARD-1 expression dominates in phytohemagglutinin-activated cells [14].

We found that in both healthy children and children with AIM, DR3 expression on the surface of live freshly isolated nTh and nCTL was very low or even absent (Figures 4 and 5). Apoptosis of naive T-lymphocytes was accompanied by a dramatically increasing density of DR3 expression on their surface. Thus, increased surface density of DR3 expression can be considered to be a marker of apoptosis of naive T-lymphocytes. 
In healthy children, when apoptosis developed in control $\mathrm{nTh}$, the percentage of live DR3 ${ }^{-}$cells decreased and the percentage of live DR $3^{+}$nTh did not change. The density of DR3 expression on the surface of live cells grew up. Thus, among nTh, DR3 ${ }^{-}$cells preferentially die by apoptosis, and the number of DR3 ${ }^{+}$ $\mathrm{nTh}$ is maintained at a constant level. The same pattern was observed in anti-DR3 $\mathrm{mAb}$-treated $\mathrm{nTh}$, but in contrast to the control, there was no increase of DR3 expression detected on the membrane of live cells. Also DR3 stimulation led to the reduction of the percentage of DR3 ${ }^{-} \mathrm{nTh}$ in early stage of apoptosis, which was not observed in the control. Meanwhile, the percentage of DR $3^{+}$early apoptotic nTh did not change. We believe that live DR3-expressing nTh can participate in the regulation of apoptosis of nTh that does not express this receptor. As the result, in nTh, DR3 activation can inhibit proapoptotic activity of $\mathrm{DR}^{+}$cells.

In healthy children in control nCTL, the growth of apoptosis was accompanied by the reduction of the percentage of live DR $3^{+}$cells. Meanwhile, the percentage of live DR3 ${ }^{-} \mathrm{nCTL}$ stayed the same. We suppose that in healthy children, unlike nTh, among nCTL DR3-expressing cells can be more susceptible to apoptosis than DR3-negative nCTL. In contradiction to control, in anti-DR3 $\mathrm{mAb}$-treated $\mathrm{nCTL}$, the percentage of both $\mathrm{DR} 3^{-}$and $\mathrm{DR} 3^{+}$live cells decreased. Thus, like in $\mathrm{nTh}, \mathrm{DR}^{+}{ }^{+} \mathrm{nCTL}$ can be capable of regulating the apoptosis of $\mathrm{DR} 3^{-}$ cells. Unlike nTh, in nCTL DR3 function is opposite: DR3 activation can lead to the enhancement of apoptosis of DR3 ${ }^{-}$cells.

In healthy children, freshly isolated $\mathrm{nCTL}$ was characterized by the large level of apoptosis, the low percentage of live DR3- cells, the high percentage of live and early apoptotic DR $3^{+}$cells and the high DR3 MFI compared with freshly isolated $\mathrm{nTh}$. After $20 \mathrm{~h}$ of cultivating of isolated $\mathrm{nCTL}$, we found out that the percentage of live DR $3^{+}$and early apoptotic DR $3^{-}$cells and the level of apoptosis of nCTL went down to values of nTh (Figures 3 and 5). The results suggest that in healthy children, a large amount of DR $3^{+}$cells and their enhanced activity can cause the high level of apoptosis of $\mathrm{nCTL}$ compared with $\mathrm{nTh}$. We speculate that the apoptosis of nCTL in vivo can be regulated by elements of cellular microenvironment by means of DR3 activation. We also assume that DR3-mediated regulation of apoptosis can be more typical for nCTL than for $\mathrm{nTh}$.

According to the literature data, DR3 stimulation, as well as its overexpression, causes spontaneous apoptosis in cell lines of T-lymphocyte origin $[14,28]$. In our study, the stimulation of naive T-lymphocytes with anti-DR3 mAb did not lead to the change in the percentage of apoptotic cells compared with control (Figures 3 and 6a). The absence of significant changes in the level of apoptosis may be due to the paucity of DR $3^{+}$cells and the population composition of T-lymphocytes. 
Like in healthy children, in children with AIM, the apoptosis of nTh was accompanied by the reduction of the percentage of live DR3 ${ }^{-}$cells, while the percentage of live $\mathrm{DR}^{+}{ }^{+} \mathrm{nTh}$ did not change. Also, like in healthy children, we detected the decrease in the percentage of early apoptotic DR3 ${ }^{-}$cells in anti-DR3 mAb-treated nTh, but not in control cells. We suppose that, like in healthy children, in children with AIM, DR ${ }^{+}$nTh can participate in the regulation of apoptosis of DR3 ${ }^{-} \mathrm{nTh}$ and DR3 activation can lead to the inhibition of the apoptosis of nTh. Therefore, the DR3 function as a regulator of apoptosis of nTh did not change in children with AIM.

Unlike healthy children, in children with AIM, the apoptosis of control nCTL was accompanied by the decrease in the percentage of live DR3 ${ }^{-}$cells, while the percentage of live DR $3^{+}$nTh stayed at a constant level. DR3 activation gave the opposite effect: the percentage of live DR $3^{+}$nCTL decreased, while the percentage of live DR ${ }^{-}$cells did not change. Besides that, addition of anti-DR3 $\mathrm{mAb}$ resulted in a decrease in the percentage of $\mathrm{nCTL}$ in early stage of apoptosis by reducing the amount of early apoptotic DR $3^{-}$cells. Thus, in children with AIM, the stimulation of $\mathrm{nCTL}$ with anti-DR3 $\mathrm{mAb}$ led to the preferential loss of DR3 ${ }^{+}$ cells while reducing the apoptosis of DR3 ${ }^{-}$nCTL. We assume that in both healthy children and children with AIM, DR ${ }^{+}$nCTL can enhance the apoptosis of DR3 ${ }^{-}$ cells. However, in children with AIM, DR3 activation can initiate the apoptosis of $\mathrm{DR}^{+} \mathrm{nCTL}$, which in its turn causes the softening of their proapoptotic influence on the other cells.

In children with AIM, no difference was found in the level of apoptosis or DR3 expression pattern between freshly isolated nTh and nCTL. In children with AIM, the level of apoptosis of freshly isolated naive T-lymphocytes did not differ in nTh but was low in nCTL compared with healthy children. Also in children with AIM, the percentage of live DR3 ${ }^{-}$cells did not differ in nTh but was greater in nCTL than in healthy children (Figures 3 and 5). DR3 function in the regulation of apoptosis of naive T-lymphocytes did not change in children with AIM: DR3 activation inhibits apoptosis of nTh and enhances apoptosis of nCTL. However, in children with AIM, the enhanced susceptibility of regulatory live DR3 ${ }^{+}$nCTL to DR3-mediated apoptosis resulted in a significant reduction of the apoptosis of the total pool of nCTL compared with healthy children.

It is shown that, depending on the conditions and the type of cell, the activation of death receptors can lead to a cell proliferation and an activation of inflammatory reactions as well as an initiation of apoptosis. Signaling outcome depends on the type of ligand, the set of adapter proteins and signaling systems, and the internalization of the receptor as an important event in the process of outcome regulation [29]. According to our data, DR3 was able to regulate the apoptosis of naive T-lymphocytes both in healthy children and in children with 
AIM. In healthy children, DR3 activation led to increased apoptosis of nCTL and in children with AIM, on the contrary, to its suppression. In nTh, both in healthy children and children with AIM, DR3 exhibits anti-apoptotic properties. DR3mediated regulation of apoptosis of naive T-lymphocytes in children with AIM can be considered as one of the mechanisms of changing the CD4/CD8 T-lymphocytes ratio toward the significant increase in virus-specific $\mathrm{CD} 8^{+}$ T-cells [30]. Thus, DR3 can play a significant role in increasing the amount of cytotoxic T-lymphocytes and thus in the elimination of the virus.

\title{
Conflict of Interest
}

\author{
None declared.
}

\section{References}

1. Womack, J., Jimenez, M.: Common questions about infectious mononucleosis. Am Fam Physician 91, 372-376 (2015).

2. Tanner, J. E., Alfieri, C.: Epstein-Barr virus induces Fas (CD95) in T cells and Fas ligand in B cells leading to T-cell apoptosis. Blood 94, 3439-3447 (1999).

3. Balfour, H. H., Dunmire, S. K., Hogquist, K. A.: Infectious mononucleosis. Clin Transl Immunol 4, e33 (2015).

4. Balfour, H. H., Odumade, O. A., Schmeling, D. O., Mullan, B. D., Ed, J. A., Knight, J. A., Vezina, H. E., Thomas, W., Hoqquist, K. A.: Behavioral, virologic, and immunologic factors associated with acquisition and severity of primary Epstein-Barr virus infection in university students. J Infect Dis 207, 80-88 (2013).

5. Hislop, A. D., Taylor, G. S., Sauce, D., Rickinson, A. B.: Cellular responses to viral infection in humans: Lessons from Epstein-Barr virus. Annu Rev Immunol 25, 587-617 (2007).

6. Palendira, U., Low, C., Chan, A., Hislop, A. D., Ho, E., Phan, T. G., Deenick, E., Cook, M. C., Riminton, D. S., Choo, S., Loh, R., Alvaro, F., Booth, C., Gaspar, H. B., Moretta, A., Khanna, R., Rickinson, A. B., Tangye, S. G.: Molecular pathogenesis of EBV susceptibility in XLP as revealed by analysis of female carriers with heterozygous expression of SAP. PLoS Biol 9, e1001187 (2011).

7. Long, H. M., Chagoury, O. L., Leese, A. M., Ryan, G. B., James, E., Morton, L. T., Abbott, R. J., Sabbah, S., Kwok, W., Rickinson, A. B.: MHC II tetramers visualize human CD4+ T cell responses to Epstein-Barr virus infection and demonstrate atypical kinetics of the nuclear antigen EBNA1 response. J Exp Med 210, 933-949 (2013).

8. Scherrenburg, J., Piriou, E. R., Nanlohy, N. M., van Baarle, D.: Detailed analysis of Epstein-Barr virus-specific CD4+ and CD8+ $\mathrm{T}$ cell responses during infectious mononucleosis. Clin Exp Immunol 153, 231-239 (2008).

9. Kimura, M. Y., Pobezinsky, L. A., Guinter, T., Thomas, J., Adams, A., Park, J. H., Tai, $\mathrm{X}$., Singer, A.: IL-7 signaling must be intermittent, not continuous, during CD8 T cell 
homeostasis to promote cell survival instead of cell death. Nat Immunol 14, 143-151 (2013).

10. Hapuarachchi, T., Lewis, J., Callard, R. E.: A mechanistic model for Naive CD4 T cell homeostasis in healthy adults and children. Front Immunol 4, 366 (2013).

11. Mao, J. Q., Yang, S. L., Song, H., Zhao, F. Y., Xu, X. J., Gu, M. E., Tang, Y. M.: Clinical and laboratory characteristics of chronic active Epstein-Barr virus infection in children. Chin J Contemp Pediatr 16, 1081-1085 (2014).

12. Migone, T. S., Zhang, J., Luo, X., Zhuang, L., Chen, C., Hu, B., Hong, J. S., Perry, J. W., Chen, S. F., Zhou, J. X., Cho, Y. H., Ullrich, S., Kanakaraj, P., Carrell, J., Boyd, E., Olsen, H. S., Hu, G., Pukac, L., Liu, D., Ni, J., Kim, S., Gentz, R., Feng, P., Moore, P. A., Ruben, S. M., Wei, P.: TL1A is a TNF-like ligand for DR3 and TR6/DcR3 and functions as a T cell costimulator. Immunity 16, 479-492 (2002).

13. Budd, R. C.: Death receptors couple to both cell proliferation and apoptosis. J Clin Invest 109, 437-442 (2002).

14. Screaton, G. R., Xu, X. N., Olsen, A. L., Cowper, A. E., Tan, R., McMichael, A. J., Bell, J. I.: LARD: A new lymphoid-specific death domain containing receptor regulated by alternative pre-mRNA splicing. Proc Natl Acad Sci U S A 94, 4615-4619 (1997).

15. Meylan, F., Song, Y. J., Fuss, I., Villarreal, S., Kahle, E., Malm, I. J., Acharya, K., Ramos, H. L., Lo, L., Mentink-Kane, M. M., Wynn, T. A., Migone, T. S., Strober, W., Siegel, R. M.: The TNF-family cytokine TL1A drives IL-13-dependent small intestinal inflammation. Mucosal Immunol 4, 172-185 (2011).

16. Meylan, F., Davidson, T. S., Kahle, E., Kinder, M., Acharya, K., Jankovic, D., Bundoc, V., Hodges, M., Shevach, E. M., Keane-Myers, A., Wang, E. C., Siegel, R. M.: The tumor necrosis factor-family receptor DR3 is essential for diverse T-cell mediated inflammatory diseases. Immunity 29, 79-89 (2008).

17. Wang, E. C., Thern, A., Denzel, A., Kitson, J., Farrow, S. N., Owen, M. J.: DR3 regulates negative selection during thymocyte development. Mol Cell Biol 21, 3451-3461 (2001).

18. Twohig, J. P., Marsden, M., Cuff, S. M., Ferdinand, J. R., Gallimore, A. M., Perks, W. V., Al-Shamkhani, A., Humphreys, I. R., Wang, E. C.: The death receptor 3/TL1A pathway is essential for efficient development of antiviral $\mathrm{CD}^{+}$and $\mathrm{CD} 8^{+} \mathrm{T}$-cell immunity. FASEB J 26, 3575-3586 (2012).

19. R Development Core Team: R: A language and environment for statistical computing. R Foundation for Statistical Computing, Vienna, Austria, 2009, p. 409.

20. RStudio Team: RStudio: Integrated Development for R. RStudio, Inc., Boston, MA, 2015.

21. Gastwirth, J. L., Gel, Y. R., Hui, W. L. W., Lyubchich, V., Miao, W., Noguchi, K.: Lawstat: An R package for biostatistics, public policy, and law. Version 2.4.1., 2013, https://cran. r-project.org/web/packages/lawstat/index.html.

22. Hothorn, T., Bretz, F., Westfall, P.: Simultaneous inference in general parametric models. Biom J 50, 346-363 (2008).

23. Le Meur, N., Hahne, F., Ellis, B., Haaland, P.: FlowCore: Basic structures for flow cytometry data. R package version 3.1.1., 2014, http://bioconductor.org/packages/release/ bioc/html/flowCore.html.

24. Pinheiro, J., Bates, D., DebRoy, S., Sarkar, D., R Core Team: nlme: Linear and nonlinear mixed effects models. R package version 3.1-117, 2014, https://cran.r-project.org/web/ packages/nlme/index.html. 
25. R Core Team: R: A language and environment for statistical computing. R Foundation for Statistical Computing, Vienna, Austria, 2014.

26. Wickham, H.: ggplot2: Elegant graphics for data analysis. Springer, New York, NY, 2009.

27. Jones, G. W., Stumhofer, J. S., Foster, T., Twohig, J. P., Hertzog, P., Topley, N., Williams, A. S., Hunter, C. A., Jenkins, B. J., Wang, E. C., Jones, S. A.: Naive and activated T cells display differential responsiveness to TL1A that affects Th17 generation, maintenance, and proliferation. FASEB J 25, 409-419 (2011).

28. Utkin, O. V., Novikov, V. V.: Death receptors in modulation of apoptosis. Biol Bull Rev 132, 381-390 (2012).

29. Schneider-Brachert, W., Heigl, U., Ehrenschwender, M.: Membrane trafficking of death receptors: Implications on signalling. Int J Mol Sci 14, 14475-14503 (2013).

30. Sulik, A., Oldak, E., Kroten, A., Lipska, A., Radziwon, P.: Epstein-Barr virus effect on frequency of functionally distinct $\mathrm{T}$ cell subsets in children with infectious mononucleosis. Adv Med Sci 59, 227-231 (2014). 